\title{
Urban legends in gait analysis
}

\author{
Paul Allard ${ }^{1, *}$, Sébastien Leteneur ${ }^{2}$, Éric Watelain ${ }^{2}$, and Mickaël Begon ${ }^{1}$ \\ ${ }^{1}$ Department of Kinesiology, University of Montreal, C.P. 6128, succursale Centre-ville, Montréal (Québec), H3C 3J7, Canada \\ ${ }^{2}$ Université Lille Nord de France, Lille, France ; UVHC, LAMIH, Universite de Valenciennes, France ; CNRS, UMR CNRS 8201, \\ Département SHV, Valenciennes, France
}

Received 19 April 2016, Accepted 7 February 2017

\begin{abstract}
Impaired gait patterns are often compared to that of a group of able-bodied subjects. This latter group usually forms a gold standard. Variability in able-bodied gait parameters could reflect different normal locomotion patterns. Furthermore, a lack of statistical difference between patient data and control subjects can hide a perturbed gait pattern. This review on normal gait patterns discusses three well-accepted hypotheses, which can be called into question. These are that we all walk alike, gait is symmetrical and propulsion comes from the foot at push-off. This diversity in normal able-bodied locomotion patterns suggests different movement strategies making it difficult for an eventual comparison with pathological gait conditions.
\end{abstract}

Keywords: gait, asymmetry, pattern, propulsion, control

Résumé. Légendes urbaines sur l'analyse de la marche. Lors de son étude, la marche pathologique est souvent comparée à celle d'un groupe de sujets sains. Ces derniers forment habituellement la référence de comparaison ou l'étalon de mesure. La variabilité dans les données des sujets témoins peut être la conséquence de la présence de plusieurs patrons de marche sensiblement différents. Ainsi, une absence de différence statistique entre les patients et les sujets témoins peut cacher une démarche pathologique. La revue de littérature réalisée sur les patrons de marche discute trois hypothèses généralement admises, mais qui peuvent être remises en cause. Celles-ci sont que le patron de marche normale est identique pour tout le monde, la marche est symétrique et la propulsion provient du pied lors de la poussée. Il semble que la diversité dans la marche des sujets sains suggère différentes stratégies de locomotion, ce qui pourrait limiter une comparaison éventuelle avec des conditions pathologiques.

Mots clés : locomotion, asymétrie, patron de marche, propulsion, contrôle

\section{Introduction}

The gait pattern of individuals with impaired locomotion due to an injury, a physical disability or a neurological disorder is often compared to that of able-bodied subjects. The latter usually forms a gold standard. Since Marey (1873) and Muybridge (1979), much effort has been devoted to quantify able-bodied gait and establish a gold standard. Differences between pathological gait parameters and those of a gold standard identify where the musculoskeletal disorder lies while its absence usually suggests normality. Nonetheless, variability exists in the gait parameters of a control group representing the gold standard. It is often associated with a large but normal distribution of an otherwise natural phenomenon. When the mean values of the control and patient groups are alike,

\footnotetext{
*Corresponding author: paul.allard@umontreal.ca
}

a large variability in either group can hide a perturbed gait pattern. Similarly, large variabilities in both groups could also eliminate a statistical difference even when the mean values are somewhat far apart (Grau et al., 2008).

Measurement errors from the type and make of instruments, models and walking speed could account for differences observed between studies. However, it does not explain those observed within the same study. Another source of variability comes from a limited number of gait trials to characterize a single subject. Following the analysis of 512 gait strides, Bollens, Crevecoeur, Detrembleur, Guillery and Lejeune (2012) concluded that characteristics of long-range autocorrelations in stride duration variability are constant over a large range of gait speeds and invariant in populations of different ages. Though, their approach is convenient to assess stride duration because it reflects the walking tempo and is considered as a clinical outcome measure (Bollens, 
Crevecoeur, Nguyen, Detrembleur \& Lejeune, 2010), it could be difficult to apply to time varying parameters such as joint reaction forces and moments. Nonetheless, the question of using a single gold standard remains open.

The questions to be addressed in this review stem from three well-accepted but still controversial hypotheses applied to able-bodied gait analysis. These are that:

- we all walk alike;

- gait is symmetrical;

- propulsion comes from the foot at push-off.

Though there are other simplifying hypotheses when performing a gait analysis such as limbs are considered rigid segments, those selected are rather controversial and test the rational for having a single gold standard for comparing gait patterns. The methodological approach is that of a narrative review where selected papers are compared and summarized on the basis of the authors' experience. The objectives of this brief review are to bring up points of disagreement and raise questions to highlight the drawbacks of using a single gold standard to characterize able-bodied gait and stimulate an interest in developing reference groups of subjects with different normal walking patterns.

\section{Do we all walk alike?}

Though everybody appears to walk the same way, one can recognize someone by her or his gait. Already in 1830, Balzac (2012) described the atypical gait of sailors, soldiers and politicians. Since then, distinct locomotion patterns grouped into clusters or gait families were shown to exist in bodied gait (Vardaxis, Allard, Lachance, \& Duhaime, 1998) as well as in running (Phinyomark, Osis, Hettinga, \& Ferber, 2015). This diversity in normal ablebodied locomotion patterns suggests different movement strategies making it difficult to compare them to pathological gait conditions. This comparison is further complicated by different gait clusters within similar disabled individuals (Roche, Pradon, Cosson, Robertson, Marchiori, \& Zory, 2014) thus weakening the rational for a single gold standard (Phinyomark et al., 2015).

Different gait clusters reflect a distinctive pattern of locomotion and not compensatory actions taken to maintain a unique way of walking. Using three-dimensional (3-D) muscle powers developed at the joints of the right lower limb, Vardaxis et al. (1998) have shown that more than one pattern exists in able-bodied gait. The first cluster of young adults was characterized by subjects propelling themselves with a strong hip pull and ankle push (Allard, Lachance, Aïssaoui \& Duhaime, 1996). The second and third families showed a gradual increase in the peak hip muscle power at heel-strike and a reduction of the natural walking speed. The fourth and fifth clusters used sagittal hip muscle activity at heel-strike to propel themselves. These last two strategies were considered less efficient because their walking speed was the slowest. Though walking speed has a significant influence on joint kinematics (Schwartz, Rozumalski \& Trost, 2008) and could account for differences between the last two clusters and the other three, it does not explain the cluster segregation where speed is similar.

Using a similar approach, Watelain, Barbier, Allard, Thevenon and Angué (2000) also performed a cluster analysis to differentiate the gait patterns between young and elderly men. Their hierarchical gait classification is shown in Figure 1. The dendogram reads from the bottom, starting with each individual gait performance based on a set of gait data comprising of phasic and temporal parameters as well as 3-D peak power values of the right lower limb. A single gait performance is then grouped with another having similar characteristics. Interestingly, the gait trials of the same subject were generally grouped together. In order words, the global gait features of a subject are his own and therefore could be recognizable from other individuals.

A family of trials or subjects is obtained when the within cluster variability is reached (Chen \& Shiavi, 1990). Watelain et al. (2000) were able to distinguish the gait characteristics of young (28 years) men from an older population (62 years) but also reported different family clusters in both young and old individuals as shown in Figure 1. Since their objectives were to distinguish the gait patterns of these populations and to determine whether elderly subjects displayed different gait patterns, the young subjects gait trials were identified as a single group though they comprised of several gait clusters. The first group of elderly subjects had the slowest walking speed and had the lowest peak muscle power values. The second family of elderly men displayed a slow walking speed due to a reduction in cadence and a slightly smaller stride length. The last group was characterized by a walking speed and muscle powers close to those of the young men. They concluded that the gait patterns of healthy elderlies are natural and do reflect any underlying musculoskeletal ailment.

These observations raise the question of what brings about different walking patterns. The trunk which accounts for more than $50 \%$ of the body mass was shown to be associated with age-related changes (Takahashi et al., 2005) and with dynamic stability control in elderly individuals (Hurt, Rosenblatt, Crenshaw, \& Grabiner, 2010). Compensatory lower limb kinematic adaptations were also reported to maintain balance during walking due to imposed trunk-flexed postures (Saha, Gard, \& Fatone, 2008).

Natural sagittal and frontal plane trunk inclinations attracted little attention because of mean values close to zero during normal gait (Leteneur, Gillet, Sadeghi, Allard, \& Barbier, 2009; Begon, Leardini, Belvedere, Farahpour, \& Allard, 2015). In a study by Leteneur et al. (2009), ablebodied subjects were grouped as forward or backward leaners according to the median of their mean sagittal trunk inclination measured during the stance phase of gait. Those with a backward trunk inclination developed strong hip flexor activity at push-off while those with a forward lean used their hip muscles throughout the stance phase. These gait strategies were associated with two of 


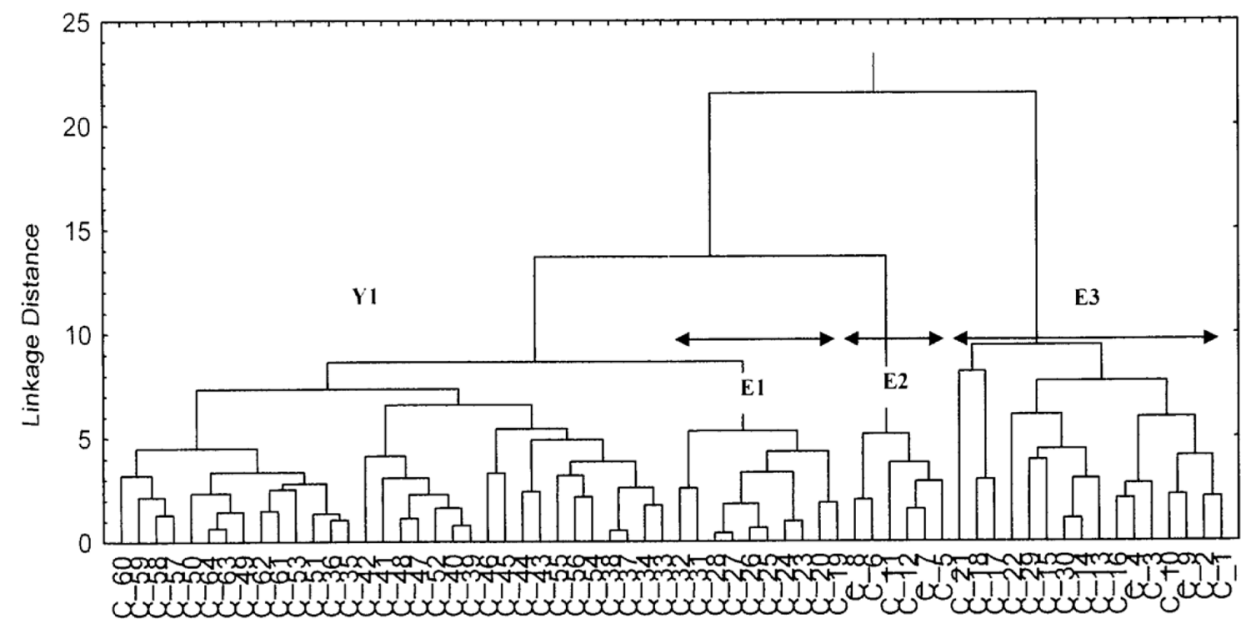

Fig. 1. Dendrogram representing the minimum variance hierarchical classification of the gait patterns of healthy young and elderly men. Four groups (families) are labeled Y1 for the young-men's family, and El to E3 for the 3 elderly-men families. The figure is read from the bottom, where the individual trials are listed, in 4 families, to top, where all the gait trials are grouped into a single family. Reproduced with permission (Watelain et al., 2000).

the family clusters described by Vardaxis et al. (1998). Leardini, Berti, Begon and Allard (2013) reported for 30 able-bodied subjects a mean sagittal trunk inclination range of $20^{\circ}$ during gait. After dividing the subjects according to the mean sagittal trunk inclination, they observed notable differences in the thorax-to-pelvis motion, shoulder-to-thorax and thorax-to-laboratory movement as well as modifications in the sagittal trunk inclinations and in lateral bending motions. They concluded that sagittal thorax inclinations modified the 3-D kinematic patterns of the upper trunk segments during natural gait. Sagittal trunk inclination could then be responsible, at least in part, to the variability in the way we walk.

Although sagittal trunk inclinations alter body kinematics, little is known about the extent of frontal trunk lean during gait and its relation to sagittal trunk orientation. Using the same approach for dividing normal subjects according to the median value, Begon et al. (2015), tested the effects of lateral trunk attitudes on the 3 -D kinematics of the shoulder, thorax and pelvis motions as well as the interaction between trunk lateral lean on the mean forward/backward thorax inclinations. As opposed to sagittal mean trunk inclination during gait, the range of trunk lateral lean did not modify upper body 3-D kinematics. However, the leaning side was shown to perturb thorax 3-D rotations throughout the gait cycle and pelvic rotation during body weight transfer from the leading to the trailing limb. The authors argued that preferred trunk lateral lean combined to thorax and pelvic rotations could be an exacerbating factor in falling.

Variability in the individual gait parameters of a normal population are considered as fine dynamic postural adjustments. These can be attributed in part to the trunk sagittal inclination or the shoulder, thorax and pelvic kinematic interactions. However, when these variabilities observed in different lower body segments are grouped together and considered as a set of information rather than individual parameters, different able-bodied gait patterns appear to emerge.

\section{Is gait symmetrical?}

Gait symmetry implies that the trailing limb's dynamic behavior is similar to that of the leading limb. One could argue that symmetrical limb motion is associated with the highly repetitive and synchronized locomotion of robots. If the counter argument is an asymmetrical gait, then one could claim that we would walk in circles. However gait asymmetries could reflect the different functional role of each limb to direct the whole body straight forward.

The debate about gait symmetry has been well documented in a review article by Sadeghi, Allard, Prince and Labelle (2000). They concluded that gait asymmetry could reflect normal adaptations between the limbs rather than consequences of a musculoskeletal disability or injury. They also proposed that each of the lower limbs contributes in varying ways to propulsion and control. Using muscles powers and mechanical energies developed during the stance phase of able-bodied subjects, it was found that the lower limbs contribute principally to either propulsion or control (Sadeghi, Sadeghi, Allard, Labelle, \& Duhaime, 2001a). Figure 2 illustrates the loading component of the leading right limb where strong hip actions during heel-strike and push-off are present. The left hip though active at heel-strike did not contribute significantly at push-off. For the knee, it was the trailing limb that dominated by its controlling actions throughout the stance phase.

The different functions of the lower limbs during gait could be the results of limb dominance. If the dominant limb is preferred for mobilization actions, then it is reasonable to assume that greater forces are exerted by 

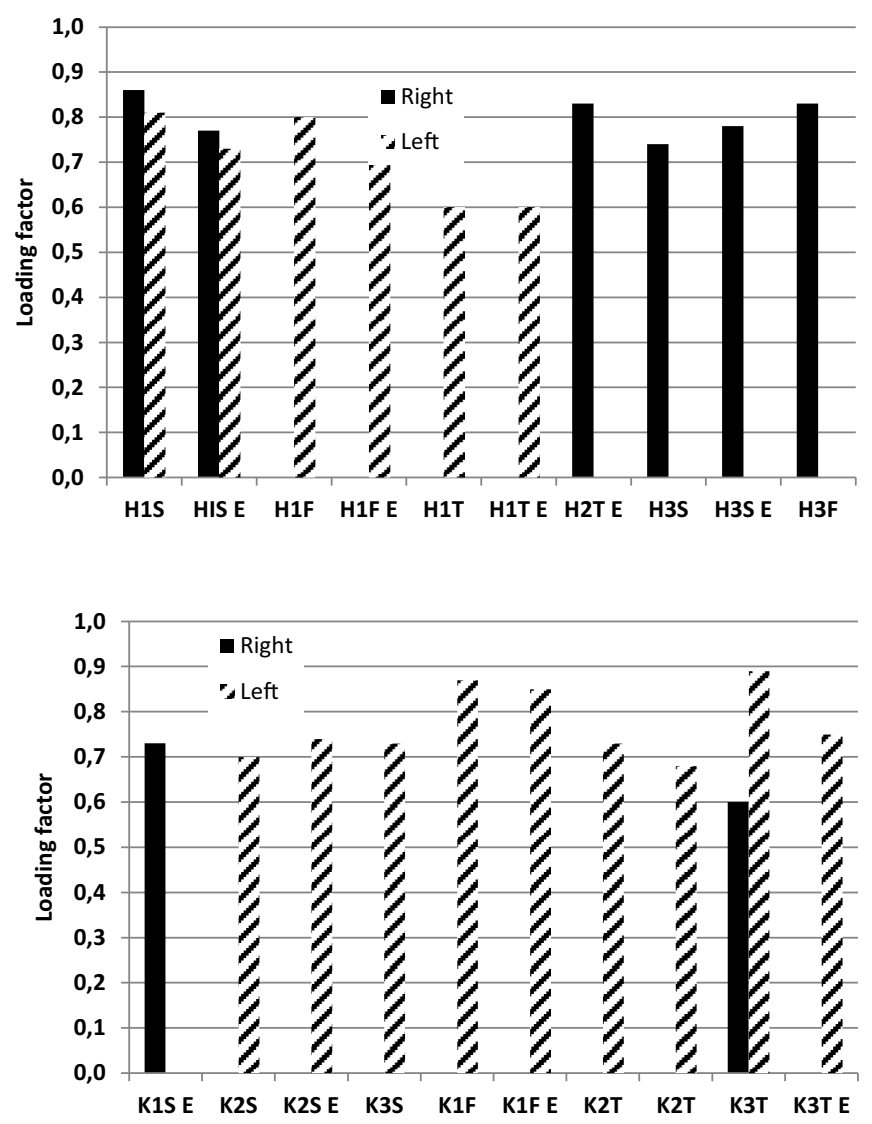

Fig. 2. Loading component values of a canonical correlation analysis for muscle powers and energies (E) calculated of the right and left hips $(\mathrm{H})$ and knees $(\mathrm{K})$ near heel-strike (1), midstance (2) and push-off (3) in the sagittal (S), frontal (F) and transverse (T) planes. Adapted from Sadeghi et al. (2001a).

this limb. This could be verified by measuring the morphology of the knee articular cartilage. Interestingly, side differences in knee cartilage volume, thickness and joint surface areas were not correlated with functional limb force estimated by an 11-item behavioral inventory test (Eckstein, Müller, Faber, Englmeier, Raiser, \& Putz, 2002). However, they were positively correlated with side differences in thigh and calf muscle cross sectional areas. Thus muscle strength imbalance between limbs could lead to different kinematic and kinetic patterns resulting in gait asymmetry.

Lower limb interaction during gait is well known. A task-specific neural interlimb coupling mechanism is assumed for an effective performance in a number of functional activities including gait (Dietz, Horstmann, \& Berger, 1989). Following a stroke, the neural interlimb coupling reorganized itself by strengthening the pathways from the unaffected part of the brainstem/hemisphere to the affected limb to compensate the sensorimotor deficit (Dietz \& Schrafl-Altermatt, 2016). One can postulate that this neural interlimb coupling adjustments normally occurs during able-bodied gait, but at a smaller scale. Using muscle powers and energies as gait parameters, the in-between lower limb data set interaction was 0.83
(Sadeghi et al., 2001b). This indicates how different actions taken by each limb are complementary towards forward walking rather than antagonistic to each other.

Though individual kinematic and kinetic differences between the lower limbs in healthy and young adults could be small, one has to consider them as a characteristic that simultaneously applies to several joints. Asymmetries were reported not only in walking but also in running. It was reported that footwear modified the afferent sensory feedback to the neuromuscular system leading to a decrease in running pattern asymmetries (Hoerzer, Federolf, Maurer, Baltich, \& Nigg, 2015). These neurophysiological processes coordinate multiple motor outputs (Rossignol, Dubuc, \& Gossard, 2006) to minimize energetic cost and predict average behavior but not the variability of repeated movements such as walking (Dingwell \& Cusumano, 2010). Variability can be associated with the neuromuscular control and regulation but it does not explain its purpose. Each individual step requires dynamic adaptations from the contralateral limb (Bohnsack-McLagan, Cusumano, \& Dingwell, 2016) leading to lower limb asymmetries.

\section{Do we push-off for propulsion or not?}

To walk, we need to generate propulsive forces and the ankle of the weight-bearing limb is assumed to fulfill this function (Winter, 1991). The peak electromyographic activity of the gastrocnemius and the soleus muscles occurring at push-off when the vertical ground reaction force reaches a maximum value has fostered this belief (Perry, 1992). If so, then how do lower limb amputees walk or run? Perry (1992) considers that the foot is free to plantar flex because the ground reaction force is acting under the metatarsal heads and not at the toes. The force in the gastrocnemius and the soleus muscles then flexes the knee. At the same time, the knee extensor (quadriceps) restrains knee motion and flexes the hip with the iliacus to flex the thigh to step forward. Since then, the controversy still exists.

Ankle push-off and hip pull strategies were further investigated by Allard et al. (1996). In a simultaneous bilateral 3-D gait analysis, they observed that the ankle contributed to $34 \%$ of the total positive work developed in the sagittal plane while the hip accounted for $56 \%$ of power for forward progression of the leading limb. The hip and ankle muscle moments developed throughout the stance phase were also analyzed to identify their main contribution to support and propulsion tasks. Results shown in Figure 3 represent the first two principal components (PC) of the hip and ankle moments developed in the sagittal plane. The first PCs accounted for $70 \%$ and $50 \%$ of the variance of the hip and ankle respectively. Generally, the significant loading component $(>0.70)$ of the first PC of the hip occurred between $5 \%-35 \%$ (extensors) and between $35 \%$ and $45 \%$ (flexors) of the stance phase. The hip moments seem to contribute largely to the advancing limb throughout the stance phase as previously described (Vardaxis et al., 1998). The contribution of the ankle plantar flexion moments were found during midstance. In 
the second PC curves which explained less than $20 \%$ of the variability, both the ankle and hip moments were significant at heel-strike and push-off. It appears that not only the ankle plantar flexors do not largely contribute to propulsion but its main contribution occurs during single limb balance to ensure stability and control of the weight-bearing limb as explained by Perry (1991).

The hip or thigh contribution to propulsion was verified by Gillet et al. (2003) by calculating the contribution of accelerated body masses of the trunk and

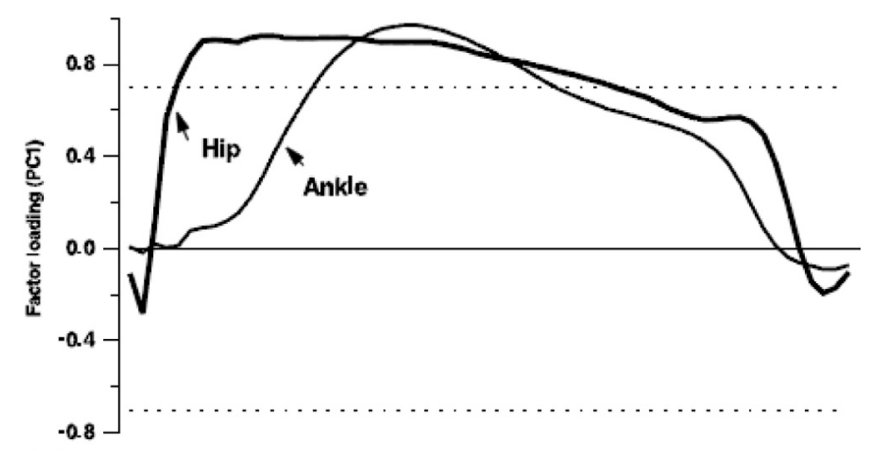

(a)

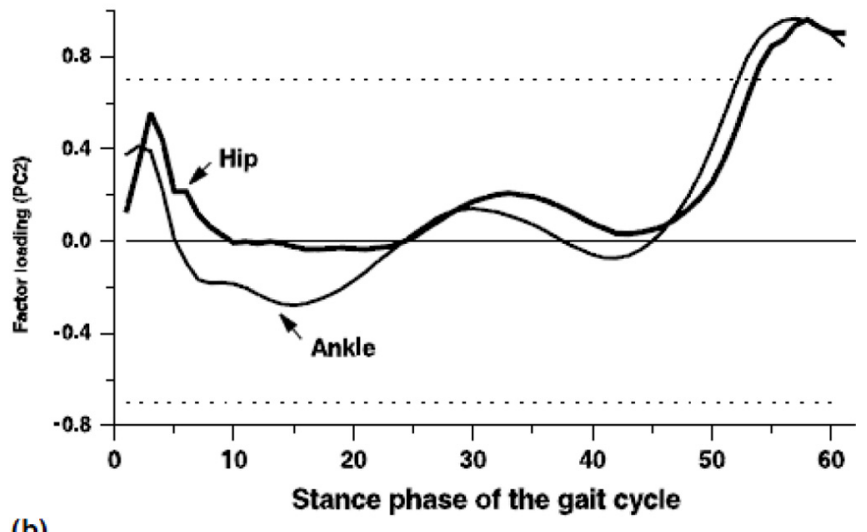

(b)

Fig. 3. First two representative curves (PC) for the ankle and hip moments. PC stands for Principal Component. Reproduced with permission (Sadeghi et al., 2001b). lower limb segments to the generation of the ground reaction forces. They reported that thighs contributed the most to the production of external forces of the lower limbs for forward progression during able-bodied gait. The controlling action of the plantar flexors hypothesis is also supported by Honeine, Schieppati, Gagey, \& Do (2013). They suggest that the triceps surae supports the body during walking and restraining it from falling rather than being responsible for the generation of propulsive force. This observation came by testing the effects of varying the propulsive force with a load of about $30 \%$ of body weight attached to the subject's abdomen while maintaining a constant progression velocity.

Figure 4A represents typical vertical ground reaction force patterns exceeding body weight and measured under the foot during gait. The double peaked shape curves reach about $800 \mathrm{~N}$ shortly after heel-strike and at push-off. The last peak is usually associated with the ankle plantar flexor activity at push-off (Winter, 1991) according to Newton's third law of motion (action/reaction). Variations in this peak force were related to healthy and disabled gait due to orthopedic or neurologic disorders (Meurisse, Dierick, Schepens, \& Bastien, 2016) as well as to running speed (Cappellini, Ivanenko, Poppele, \& Lacquaniti, 2006; Clark, Ryan, \& Weyand, 2014). However, Gillet et al. (2003) have shown that the ground reaction forces are related to Newton's second law of motion. Not only is the pattern of the vertical ground reaction force is dependent on the acceleration of whole body mass (Barbier, Allard, Guelton, Colobert, Godillon-Maquinghen, \& Lepoutre, 2003) but also on the sum of all the body segments' accelerated mass (Aissaoui, Allard, Junqua, Frossard, \& Duhaime, 1996).

The vertical ground reaction forces exceeding body weight of the same subject as in Figure 4A during a cranking motion of the contralateral limb are presented in Figure 4B. The vertical force patterns are quite similar with a coefficient of correlation above 0.9 . This simple test demonstrates that the typical vertical ground reaction forces developed during normal walking can be reproduced by a swinging limb of a person standing with a still
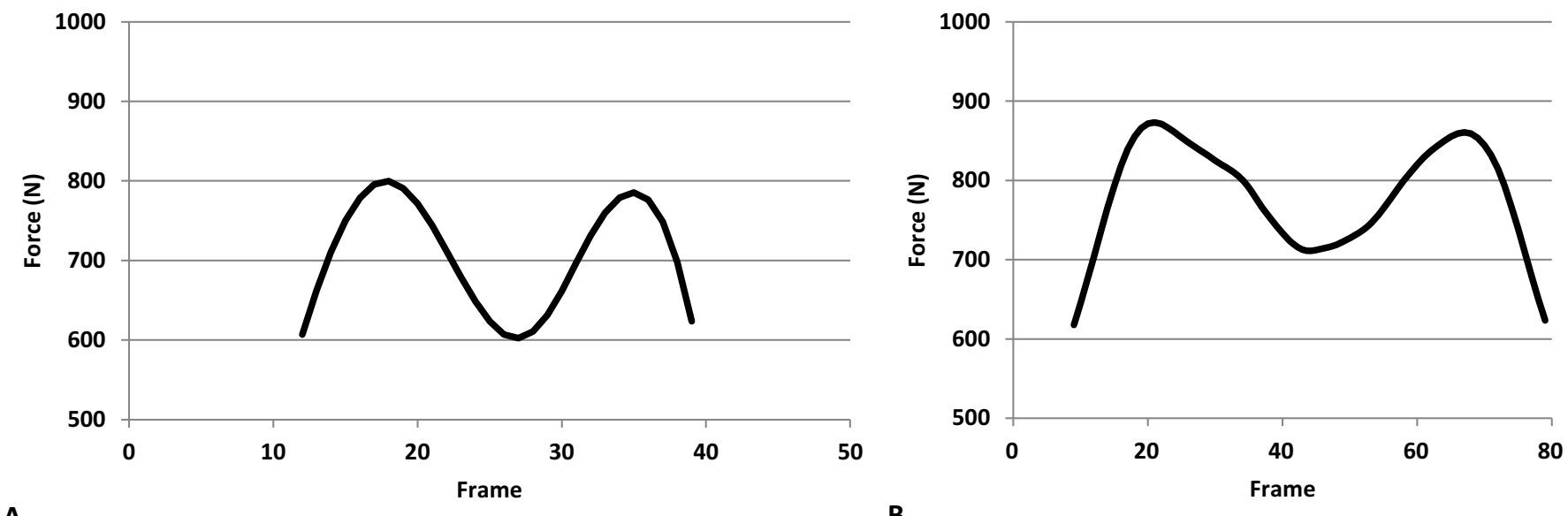

B

Fig 4. A. Vertical ground reaction forces exceeding body weight and measured during able-bodied gait and B. while the contralateral limb is swinging in a cranking motion while the same subject is standing with a motionless weight-bearing limb (right). 
weight-bearing limb. The measured resultant forces are essentially the sum of the accelerated mass of the swinging lower limb segments, if one assumes that the vertical accelerations of the body center of mass are small.

Using different parameters and various statistical means, normal gait is shown to be a complex and varied phenomenon. A better understanding could provide a greater insight into gait patterns, lower limb control and propulsion that could lead to improve rehabilitation, orthopedic, neurologic treatments as well as to novel technical aids to the benefit of the physically impaired individuals.

\section{Conclusion}

It appears that the global walking features of a subject are his own but differ from others though individuals can be grouped in clusters having similar gait characteristics. Also, more than a single walking strategy exists in both young and elderly men. These could be related to both mean sagittal and frontal trunk lean. Not only the lower limbs contribute in varying ways to propulsion and control resulting in gait asymmetry but also there is an in-between lower limb interaction to ensure global forward progression. Each individual step requires dynamics adaptations from the contralateral limb manifested by kinematic and kinetic differences in the lower limbs of healthy and young adults. Lower limb propulsion could be generated at hip while the ankle plantar flexors ensure stability and control of the weight-bearing limb. Variation in vertical ground reaction forces is largely dependent on the acceleration of the whole body center of mass and the sum of all the accelerated body segments as expressed by Newton's second law of motion.

Acknowledgements. The authors are grateful to Dr. Arne Lundberg, from the Karolinska Institute, Stockholm, Sweden on his suggestions on authoring this paper (Lundberg et al., 2014).

\section{References}

Aissaoui, R., Allard, P., Junqua, A., Frossard, L., \& Duhaime, M. (1996). Internal work estimation in 3-D gait analysis. Medical and Biological Engineering and Computing, 34, 467471.

Allard, P., Lachance, R., Aïssaoui, R. \& Duhaime, M. (1996). Simultaneous bilateral 3-D gait analysis. Human Movement Science, 15, 327-346.

de Balzac, H. (2012). Traité de la vie Élégante. Paris : Éditions Rivages.

Barbier, F., Allard, P., Guelton, K., Colobert, B., GodillonMaquinghen, A.-P., \& Lepoutre, F.-X. (2003). Estimation of the 3 -D center of mass excursion from force plate data during standing. IEEE Transaction on Neural System and on Rehabilitation Engineering, 11, 31-37.

Begon, M., Leardini, A., Belvedere, C., Farahpour, N. \& Allard, P. (2015). Effects of frontal and sagittal thorax attitudes in gait on trunk and pelvis three-dimensional kinematics. Medical Engineering and Physics, 37, 1032-1036.
Bohnsack-McLagan, N.K., Cusumano, J.P. \& Dingwell, J.B. (2016). Adaptability of stride-to-stride control of stepping movements in human walking. Journal of Biomechanics, 49, 229-237.

Bollens, B., Crevecoeur, F., Detrembleur, C., Guillery, E., \& Lejeune, T. (2012). Effects of age and walking speed on longrange autocorrelations and fluctuation magnitude of stride duration. Neuroscience, 210, 234-242.

Bollens, B., Crevecoeur, F., Nguyen, V., Detrembleur, C., \& Lejeune, T. (2010) Does human gait exhibit comparable and reproducible long-range autocorrelations on level ground and on treadmill? Gait \&6 Posture, 32, 369-373.

Cappellini, G., Ivanenko, Y.P., Poppele, R.E., \& Lacquaniti, F., 2006. Motor patterns inhuman walking and running. Journal of Neurophysiology, 95, 3426-3437.

Chen, J.J., \& Shiavi, R. (1990). Temporal feature extraction and clustering analysis of electromyographic linear envelopes in gait studies. IEEE Transactions on Biomedical Engineering, 37, 295-302.

Clark, P., Ryan, L.J., \& Weyand, P.G. (2014). Foot speed, footstrike and footwear: linking gait mechanics and running ground reaction forces. The Journal of Experimental Biology, 217, 2037-2040.

Dietz, V., Horstmann, G.A., \& Berger, W. (1989). Interlimb coordination of leg-muscle activation during perturbation of stance in humans. Journal of Neurophysiology, 62, 680-693.

Dietz, V., \& Schrafl-Altermatt, M. (2016). Control of functional movements in healthy and post-stroke subjects: Role of neural interlimb coupling. Clinical Neurophysiology, 127, 2286-2293.

Dingwell, J.B., \& Cusumano, J.P. (2010). Re-interpreting detrended fluctuation analyses of stride-to-stride variability in human walking. Gait $\&$ Posture, 32, 348-353.

Eckstein, F., Müller, S., Faber, S.C., Englmeier, K.H., Reiser, M., \& Putz, R. (2002). Side differences of knee joint cartilage volume, thickness, and surface area, and correlation with lower limb dominance - an MRI-based study. Osteoarthritis and Cartilage, 10, 914-921.

Gillet, C., Duboy, J., Barbier, F., Armand, S., Jeddi, R., Lepoutre, F.-X., \& Allard, P. (2003) Contribution of the accelerated body masses to able-bodied gait. American Journal of Physical Medicine and Rehabilitation, 82, 101109.

Grau, S., Maiwald, C., Krauss, I., Axmann, D., \& Horstmann, T. (2008). The influence of matching populations on kinematic and kinetic variables in runners with iliotibial band syndrome. Research. Quarterly for Exercise and Sport, 79, 450-457.

Hoerzer, S., Federolf, P.A., Maurer, C., Baltich, J. \& Nigg, B.M. (2015). Footwear Decreases Gait Asymmetry during Running. PLoS ONE, 10(10), e0138631. doi:10.1371/journal. pone.0138631.

Honeine, J.L., Schieppati, M., Gagey, O., \& Do, M.C. (2013) The Functional Role of the Triceps Surae Muscle during Human Locomotion. PLoS ONE, 8(1), e52943. doi:10.1371/journal. pone.0052943.

Hurt, C.P., Rosenblatt, N., Crenshaw, J.R., \& Grabiner, M.D. (2010) Variation in trunk kinematics influences variation in step width during treadmill walking by older and younger adults. Gait \& Posture, 31, 461-464.

Leardini, A., Berti, L., Begon, M., \& Allard, P. (2013). Effect of trunk sagittal attitude on shoulder, thorax and pelvis three-dimensional kinematics in able-bodied subjects during gait. PLoS ONE, 8(10), e77168. doi:10.1371/journal. pone.0077168. 
Leteneur, S., Gillet, C., Sadeghi, H., Allard, P., \& Barbier, F. (2009). Effect of trunk inclination on lower limb joint and lumbar moments in able men during the stance phase of gait. Clinical Biomechanics, 24, 190-195.

Lundberg, A., Aguilera, A., Cappozzo, A., Michaud, B., Garrido, B., Baten, C., Samnegard, E., Barbier, F., Zahalka, F., Hazime, F. A., Dalleau, G., Stylianides, G., Sadeghi, H., Boucher, J., Raso, J., Manal, K., Roren, L., Chèze, L., Eslami, M., Mathieu, M.-E., Simoneau, M., Damavandi, M., Richard, N., Salvia, P., Lacouture, P., Allard, P., Gardiner, P., Armand, S., Whitaker, T., Arndt, T., della Croce, U., Robert-Lachaîne, X., \& Begon, M. (2014). Entropy in the list of authors in scientific papers. Annals of Improbable Research, 20, 14-17.

Marey, E.J. (1873). La machine animale. Paris : Revue EPS.

Meurisse, G.M., Dierick, F., Schepens, B., \& Bastien, G.J. (2016). Determination of the vertical ground reaction forces acting upon individual limbs during healthy and clinical gait. Gait \&S Posture, 43, 245-250.

Muybridge, E. (1979) Muybridge's Complete Human and Animal Locomotion. New York: Dover.

Perry, J. (1992). Gait Analysis: Normal and Pathological Function. Thorofare: Slack Incorporated.

Phinyomark, A., Osis, S., Hettinga, B.A., \& Ferber, R. (2015). Kinematic gait patterns in healthy runners: A hierarchical cluster analysis. Journal of Biomechanics, 48, 3897-3904.

Roche, N., Pradon, D., Cosson, J., Robertson, J., Marchiori, C., \& Zory, R. (2014). Categorization of gait patterns in adults with cerebral palsy: A clustering approach. Gait $\&$ Posture, 39, 235-240.

Rossignol, S., Dubuc, R., \& Gossard, J.-P. (2006). Dynamic sensorimotor interactions in locomotion. Physiological Reviews, 86, 89-154.
Sadeghi, H., Allard, P. Prince, F. \& Labelle, H. (2000). Gait symmetry and limb dominance in able-bodied gait: A review. Gait \& Posture, 12, 34-45.

Sadeghi, H., Allard, P., Duhaime, M., \& Labelle, H. (2001a). Lower limb power relationships in bilateral able-bodied gait. American Journal of Physical Medicine and Rehabilitation, 80, 821-830.

Sadeghi, H., Sadeghi, S., Prince, F., Allard, P., Labelle, H., \& Vaughan, C.L. (2001b). Functional roles of ankle and hip sagittal moments in able-bodied gait. Clinical Biomechanics, 16, 688-695.

Saha, D., Gard, D., \& Fatone, S. (2008). The effect of trunk flexion on able-bodied gait. Gait \& Posture, 28, 653-660.

Schwartz, M.H., Rozumalski, A., \& Trost, J.P. (2008). The effect of walking speed on the gait of typically developing children. Journal of Biomechanics, 41, 1639-1650.

Takahashi T, Ishida K, Hirose D, Nagano Y, Okumiya K, Nishinaga, M., Matsubayashi, K, Doi, Y., Tani, T., \& Yamamoto, H. (2005). Trunk deformity is associated with a reduction in outdoor activities of daily living and life satisfaction in community-dwelling older people. Osteoporosis International, 16, 273-279.

Vardaxis, V., Allard, P., Lachance, R., \& Duhaime, M. (1998). Classification of able-bodied gait using 3-D muscle power. Human Movement Science, 17, 121-136.

Watelain, E., Barbier, F., Allard, P., Thevenon, A., \& Angué, J.-C. (2000). Gait pattern classification of healthy elderly men based on biomechanical data. Archives of Physical Medicine and Rehabilitation, 81, 579-586.

Winter, D.A. (1991). Biomechanics and motor control of human movement. New-York: Wiley.

Cite this article as: Allard P, Leteneur S, Watelain É, \& Begon M (2017) Urban legends in gait analysis. Mov Sport Sci/Sci Mot, $\mathbf{9 8}, 5-11$ 